\title{
Doppler Ultrasound Evaluation of Patients with Popliteal Vascular Entrapment Syndrome
}

\author{
Hai Zhong ${ }^{1}$, Yu Liang ${ }^{1}$, Guangrui Shao ${ }^{1}$, Hengtao Qi ${ }^{2}$, Yuan Zhao ${ }^{3}$, Maohua Wang ${ }^{4}$, and Wubo Yang ${ }^{1, *}$ \\ ${ }^{1}$ Department of Radiology, Second Hospital of Shandong University, Jinan, Shandong, China \\ 2Department of Ultrasound, Shandong Medical Imaging Research Institute, Jinan, Shandong, China \\ ${ }^{3}$ Shandong University of Traditional Chinese Medicine, Shandong, China \\ ${ }^{4}$ Department of Vascular Surgery, Shandong Provincial Hospital, Shandong, China
}

*Corresponding author: Wubo Yang, Department of Radiology, Second Hospital of Shandong University, Jinan, Shandong, 250033, China, Tel: 86-17660086059; E-mail: doctor1yang@163.com, 404958638@qq.com

Received: 31 Aug, 2021 | Accepted: 20 Sep, 2021 | Published: 24 Sep, 2021

Citation: Zhong H, Liang Y, Shao G, Qi H, Zhao Y, et al. (2021) Doppler Ultrasound Evaluation of Patients with Popliteal Vascular Entrapment Syndrome. J Heart Health 7(2): dx.doi.org/10.16966/2379-769X.162

Copyright: ( 2021 Zhong H, et al. This is an open-access article distributed under the terms of the Creative Commons Attribution License, which permits unrestricted use, distribution, and reproduction in any medium, provided the original author and source are credited.

\begin{abstract}
Background: To retrospectively evaluate Doppler ultrasound findings for patients with Popliteal Vascular Entrapment Syndrome (PVES).

Methods: Twenty-four patients (30 legs) who underwent surgical intervention for PVES were included in this study. The popliteal artery, popliteal vein, and surrounding musculotendinous structures were explored in all symptomatic lower extremities using ultrasound. Intraoperative findings served as the gold standard. In the absence of popliteal artery occlusion, popliteal arteries were examined using Duplex ultrasound at rest and during a provocative maneuver.

Results: Ultrasonography in 25 cases $(25 / 30,83 \%)$ showed that gastrocnemius medial head or popliteal vascular anomalies were consistent with surgical photography results. In the remaining five cases $(5 / 30,17 \%)$, no anatomical abnormalities of the popliteal fossa were discovered by the ultrasound examination. Among these, two cases were misdiagnosed as lower extremity atherosclerosis. In addition, increasing blood flow velocity was found in three cases of compressed popliteal arteries using duplex scanning during active plantar flexion, with decreasing flow at the distal end.

Conclusion: Doppler ultrasound may have a high diagnostic rate in PVES. The present study suggests that Doppler ultrasound is able to show the position of the popliteal artery and popliteal vein in an abnormal left-right alignment instead of the normal anterior-posterior alignment, as well as the medial head of the gastrocnemius muscle or an abnormal bundle that passes between popliteal artery and vein. These are crucial signs for the diagnosis of PVES. Due to its simplicity, repeatability, functional evaluation of blood flow, and non-invasiveness, ultrasound can be used as a primary screening examination modality in PVES. Provocative maneuvers could help clinicians diagnose PVES.
\end{abstract}

Keywords: Popliteal vascular entrapment syndrome; Doppler ultrasound; Provocative maneuvers

\section{Introduction}

The popliteal artery and popliteal vein normally run above the popliteal muscle between the medial and lateral heads of the gastrocnemius muscle. Popliteal Vascular Entrapment Syndrome (PVES) due to an abnormal anatomical relationship between popliteal vessels and neighboring myofascial structures results in repeated vessel compression during exercise. Young adults and active adolescents are most often affected by the syndrome, which occurs mainly in males. Claudication is the predominant clinical feature of PVES, which is characterized by calf pain after exercise [1]. After repeated irritation and compression for a long time, eventually irreversible trauma to the popliteal vessels can result in arterial stenosis, occlusion, thrombosis, post-stenotic aneurysms, and popliteal vein thrombosis [2].

Therefore, early diagnosis and treatment are crucial for decreasing morbidity and preventing complications. Imaging modalities can provide information that might allow identifying entrapment [3]. The present study described the popliteal vessel and adjacent musculotendinous structure findings by Doppler ultrasound in patients with PVES.

\section{Materials and Methods}

\section{Patients}

The local institutional ethics committee approved this retrospective study (KYLL-2019(LW)025), and the need for informed consent was waived. The present study included twenty-four patients who underwent surgical intervention for PVES between May 2011 and April 2019 in The Second Hospital of Shandong University and Shandong Provincial Hospital (Jinan, China). Six patients were affected bilaterally. A total of nineteen males and five females, ranging in age from 16 to 58 years old (mean, $32.0 \pm 12.3$ years old) were 
evaluated using Doppler sonography. This study was a retrospective study involving symptomatic patients of peripheral arterial disease. The symptom is characterized by progressive claudication, paresthesia, calf swelling, and foot coldness in persons, mostly young men. Risk factors for atherosclerosis were eliminated in all patients. Patient clinical profiles are summarized in table 1 . All patients were surgically treated.

\section{Doppler ultrasound scanning}

All subjects were analyzed with linear $(7-10 \mathrm{MHz})$ transducers connected to Philips IU 22, (Philips, Amsterdam, the Netherlands) or GE Vivid E9 (General Electric Healthcare, Milwaukee, USA). Graded dynamic assessment combined gray-scale imaging with range-gated pulsed-Doppler analysis of blood flow at each point during imaging. Subjects with relevant limbs were evaluated in a prone position by two radiologists specializing in vascular ultrasonography. Each patient was placed on an examination couch with the knee of the examined leg slightly flexed to ensure that the gastrocnemius muscle was fully relaxed. Color Doppler ultrasound of the popliteal vessel was first placed with the foot in a neutral position and then actively plantar flexed to maximum the active extension of the knee, while an assistant added resistance to the plantar flexion using manual pressure on the sole of the foot. Duplex ultrasound during this modality of plantar flexion was designated as a provocative maneuver test [4], which was performed in patients without popliteal vessel occlusion.

The neutral position was used to visualize artery occlusion, aneurysm, popliteal vessels anomalies, and presence of collateral arteries. Repeated plantar flexion until the individual becomes symptomatic may be necessary to demonstrate occlusion. Popliteal artery with a peak systolic velocity ratio exceeding $200 \%$ indicates a positive result $[5,6]$. Careful assessment of both lower limbs was required to determine if the abnormality was bilateral. Ultrasound PVES imaging was divided into six types according to Whelan and Rich classification (Table 2) $[1,7,8]$.

\section{Results}

All twenty-four patients (30 legs) underwent a successful surgical treatment, including nineteen males and five females. Popliteal vascular entrapment was presented unilaterally in eighteen patients

Table 1: Clinical profiles for patients with PVES.

\begin{tabular}{|l|c|}
\hline \multicolumn{1}{|c|}{} & Number/Total number (\%) \\
\hline Location & $10 / 24(42 \%)$ \\
\hline Left & $8 / 24(33 \%)$ \\
\hline Both & $6 / 24(25 \%)$ \\
\hline Symptoms & \\
\hline Claudication & $30 / 30(100 \%)$ \\
\hline Paresthesia & $21 / 30(70 \%)$ \\
\hline Calf swelling & $7 / 30(23 \%)$ \\
\hline Foot coldness & $8 / 30(27 \%)$ \\
\hline Risk factor & \\
\hline Exercise & $5 / 24(21 \%)$ \\
\hline Smoker & $3 / 24(13 \%)$ \\
\hline High blood pressure & $2 / 24(8 \%)$ \\
\hline
\end{tabular}

$\mathrm{N}$, number of limbs
Table 2: Types of patients with PVES.

\begin{tabular}{|l|l|}
\hline \multicolumn{1}{|c|}{ Type } & \multicolumn{1}{c|}{ Description } \\
\hline Type I & Aberrant course of the PA medial to a normal MHG \\
\hline Type II & Abnormal lateral insertion of the MHG, no deviation of PA \\
\hline Type III & Abnormal muscle bundle from MHG surrounding the PA \\
\hline Type IV & $\begin{array}{l}\text { Abnormal location of PA, deep in the popliteus muscle or a } \\
\text { fibrous band in the popliteal fossa }\end{array}$ \\
\hline Type V & $\begin{array}{l}\text { Any form of the entrapment that involves both PA and } \\
\text { popliteal vein }\end{array}$ \\
\hline Type VI & $\begin{array}{l}\text { PA is normally positioned and entrapped by a normally } \\
\text { positioned hypertrophied gastrocnemius }\end{array}$ \\
\hline $\begin{array}{l}\text { MHG indicates Medial Head of Gastrocnemius Muscle; PA: Popliteal } \\
\text { Artery }\end{array}$
\end{tabular}

and bilaterally in six patients. All cases had popliteal vessel migration or gastrocnemius medial head anomalies.

Doppler ultrasound was performed with dynamic testing in all patients. It was found that 25 cases $(25 / 30)$ of limb had an abnormal relationship between the popliteal vessel and neighboring muscular structures. The direct indication of ultrasound is an increase in the distance between the popliteal artery and the popliteal vein. Ultrasound images show the position of the popliteal artery and popliteal vein in an abnormal left-right alignment and the medial head of the gastrocnemius muscle or aberrant accessory muscle slip that passes between popliteal artery and vein (Figures 1-4). Each case was classified grounded on the findings of Doppler ultrasound. Five limbs with medial deviation of the popliteal artery were classified as type I. Twelve limbs with the medial head of the gastrocnemius origin shifting laterally were classified as type II (Figure 5). Seven limbs were type III with an anomalous accessory band of the medial head of gastrocnemius (Figure 4). One limb was type $\mathrm{V}$ with any type of entrapment that includes the popliteal vein as well as artery. All ultrasound features and classifications were confirmed by surgical photography. In the remaining five cases $(5 / 30)$, no anatomical abnormalities of the popliteal fossa were found by ultrasound examination, that is to say, no PVES was found by Doppler scan, and two of them were diagnosed as lower extremity atherosclerosis. In the other three cases, the popliteal arteries were normal in the neutral position, whereas presented with narrowed or occluded in the plantar flexed position (Table 3 and figure 6). Duplex imaging demonstrated popliteal vessel compression occlusion with blood flow interruption and significant popliteal vessel compression of the lumen. Doppler spectrum showed that the peak systolic blood flow velocity increased significantly, more than two times the value before the provocative maneuvers (Table 3). Doppler blood flow velocity was decreased near the tibiofibular stem.

When the feet were placed in the neutral position, ten of the 30 limbs with popliteal artery were stenotic (Figure 3c). Arterial occlusion was demonstrated in fifteen of the 30 limbs (Figures 2 and 4). Occlusion was characterized by hypoechoic or medially echoic thrombosis filling the lumen, no blood flow during color Doppler flow imaging, decreased blood flow velocity in the distal artery, and one-way spectrum. Partial lumen thinning was even more difficult to identify and distal segment of the popliteal artery had abundant collateral vessels. Doppler scan images also showed a post-stenotic aneurysm in one limb and a thrombosed aneurysm in another limb. With the feet placed in the plantar flexed position, unoccluded popliteal vascular flow tended to decrease or disappear. These ultrasound findings are shown in table 4. 

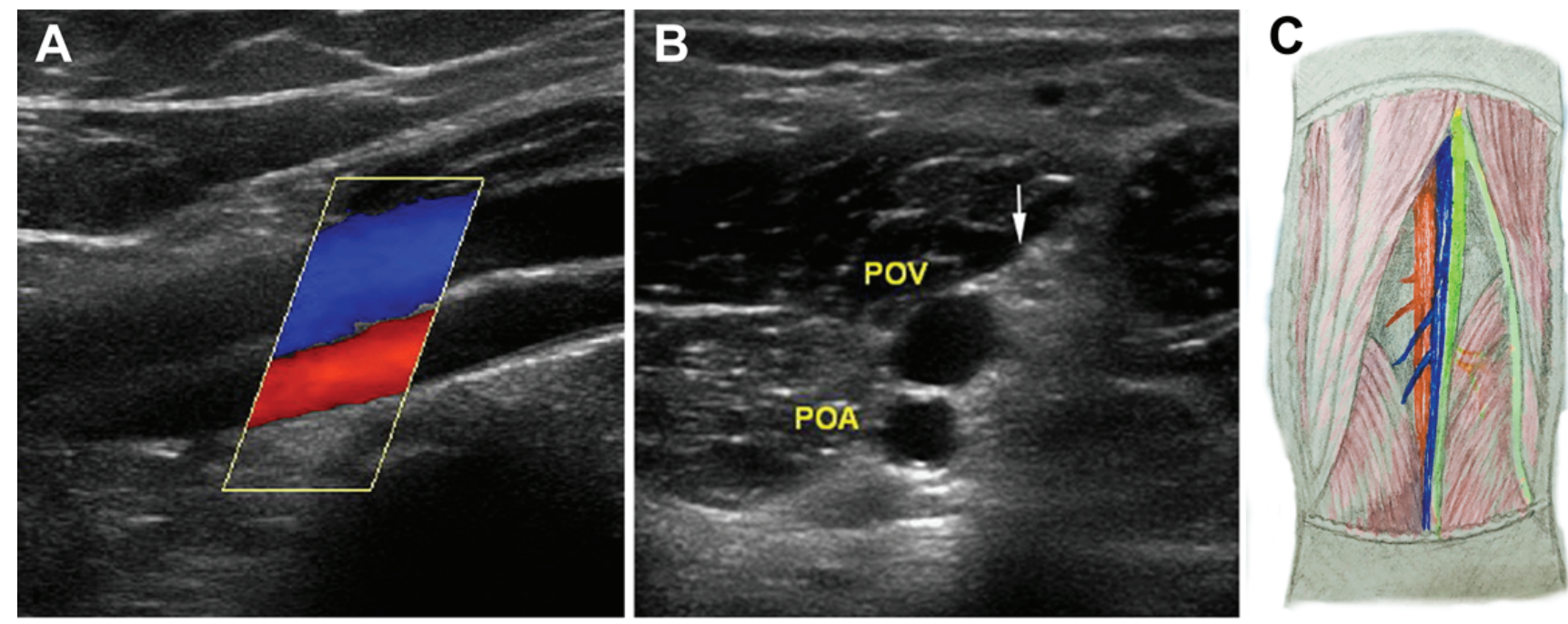

Figure 1: Normal popliteal artery, popliteal vein, and tibial nerve alignment. A: Sagittal ultrasound position shows that popliteal artery (red) and popliteal vein (blue) are in normal anteroposterior relationship; B: Transverse position shows sequence of anterior and posterior movements of tibial nerve (arrow), Popliteal Vein (POV), and Popliteal Artery (POA); C: Drawings illustrate normal popliteal vascular relationship (posterior view): front and back arrangement, followed by the tibial nerve (green), popliteal vein (blue), and popliteal artery (red) from back to front.
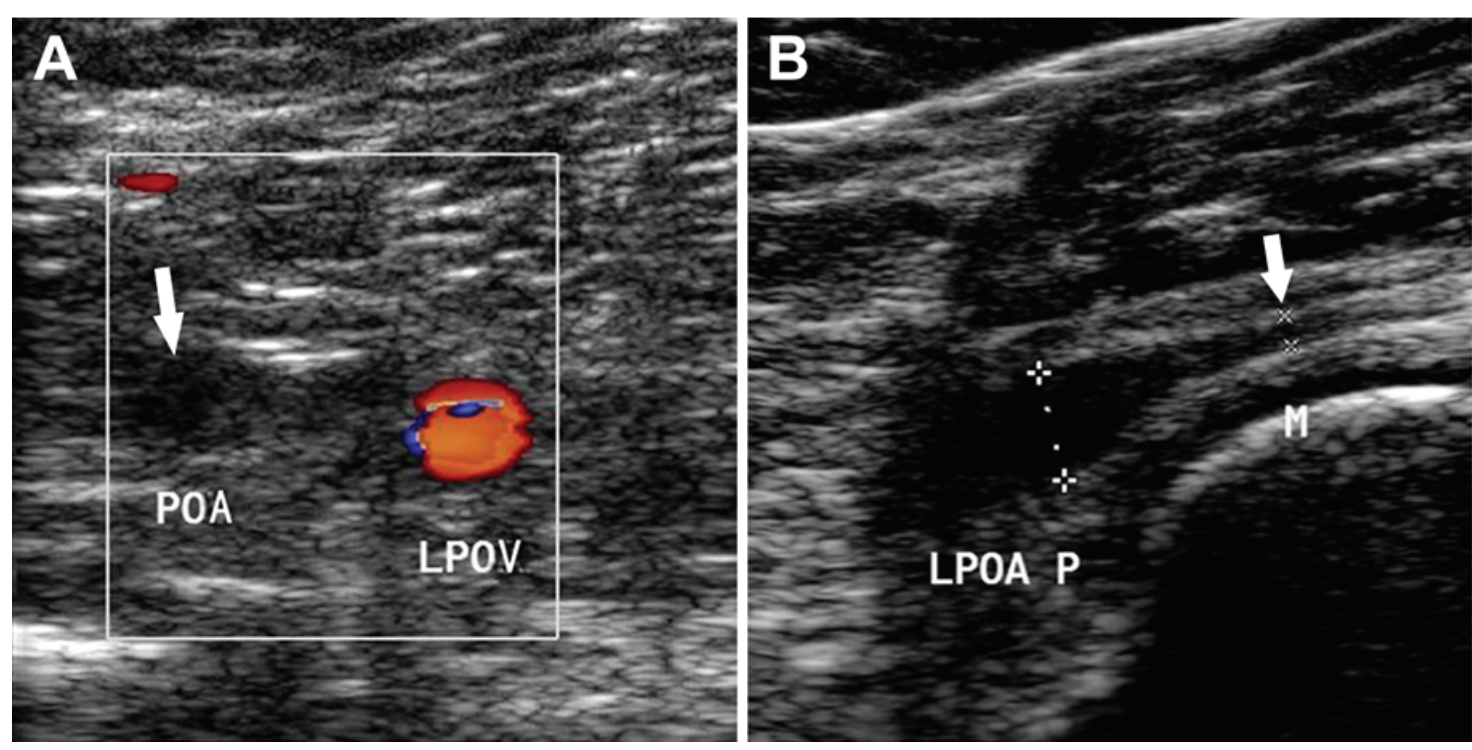

Figure 2: Left popliteal vascular entrapment syndrome in 22-year-old man who presented with intermittent claudication of left calf for three months. A: Color Doppler images for the Popliteal Artery (POA) and Popliteal Vein (POV) widening and moving from normal forward and backward orientations to left and right orientations. Left popliteal artery was occluded with hypoechoic thrombosis and no flow signal filling in the lumen (arrow); B: Proximal diameter of the left popliteal artery is within the normal range and lumen diameter of the middle and far popliteal arteries is significantly thinner (arrow).

\section{Discussion}

PVES is a rare cause of exercise-induced leg pain. Active young people and athletes without risk factors, such as smoking, heart disease, or systemic atherosclerosis, are affected. The symptoms are correlated with the degree of compression of the popliteal vessels. Popliteal vascular structure is normal in the early stage and the disease becomes evident only after vascular compression between the contracting gastrocnemius muscles occurs [9]. Repeated extrinsic vascular compression causes trauma to the vascular wall, leading to premature intrinsic atherosclerosis and thrombus formation. The pathology of PVES is considered to be progressive and resulting stenosis and turbulence can lead to post-stenotic aneurysm. In addition, acute ischemia can occur if there is an occluded artery or aneurysm with thrombosis $[2,10]$. Early diagnosis is crucial as PVES is a progressive disorder and early intervention is recommend to prevent serious complications $[9,10]$.

Color Doppler ultrasound has been widely used to diagnose peripheral vascular diseases [11-14]. This non-invasive examination 

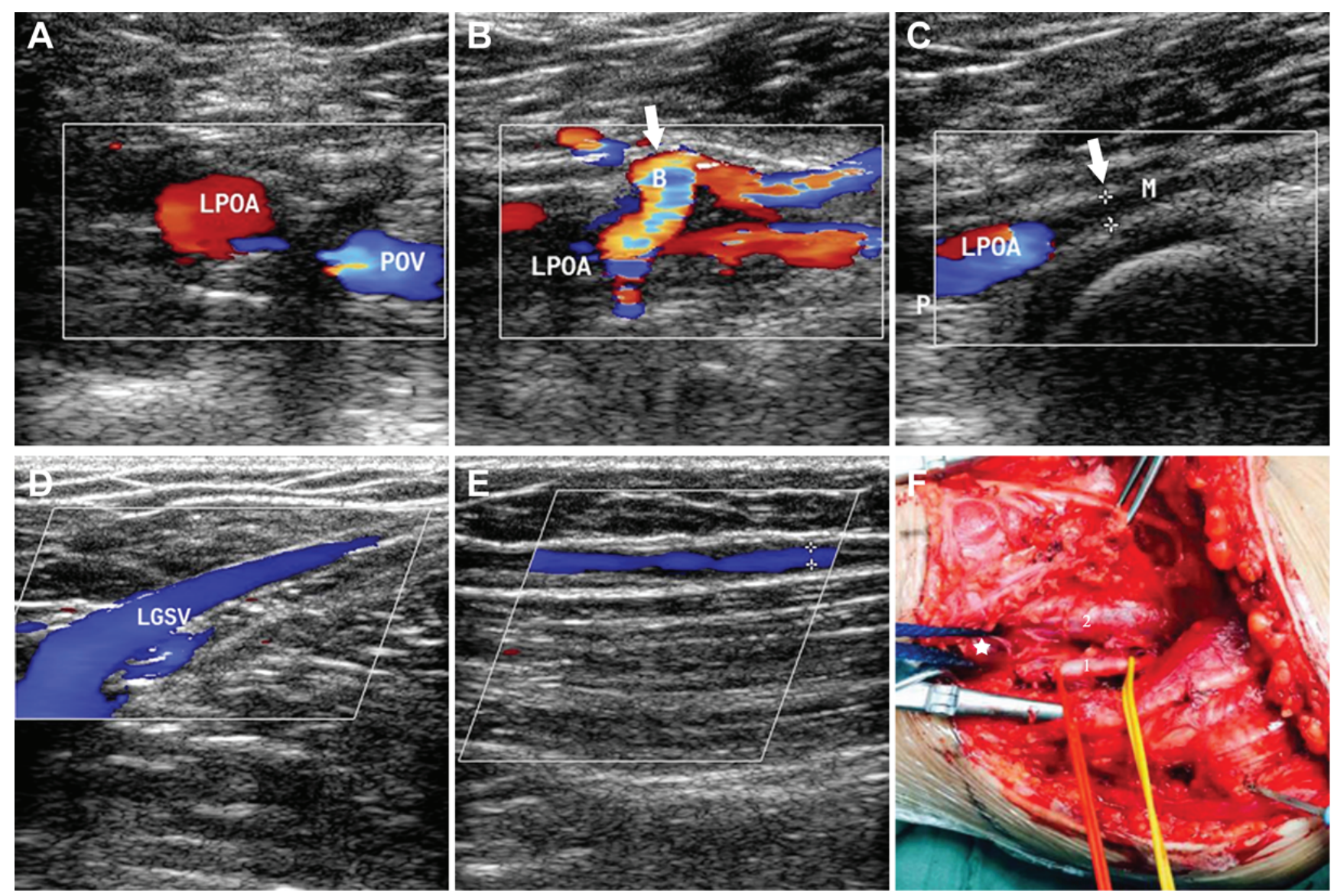

Figure 3: Left popliteal vascular entrapment syndrome in a 17-year-old man who presented with intermittent claudication and paresthesia of left lower extremity after exercise can be relieved after rest (PVES type II). A: Color Doppler images show widening of distance between the Popliteal Artery (POA) and Popliteal Vein (POV), changing from normal forward and backward alignment to abnormal left and right alignment; B: Proximal part of popliteal artery blood flow signal shows fullness and integrity, where a branch (arrow) of the popliteal artery blood vessel is detected; C: Lumen diameter of the popliteal artery in middle and far sections is significantly thinner and there is no blood flow signal in the lumen; D and E: Ultrasonic images show that blood flow signal in the Left Great Saphenous Vein (LGSV) is complete and without obvious abnormalities; F: Operation photography of left knee shows that the medial head of left gastrocnemius muscle (star) shifts outwards and downwards and lies between the popliteal artery (1) and popliteal vein (2).

does not require contrast enhancement, patient preparation before study, or radiation exposure. The present study suggests that Doppler ultrasound is able to show the position of the popliteal artery and popliteal vein in an abnormal left-right alignment instead of the normal anterior-posterior alignment, as well as the medial head of the gastrocnemius muscle or an abnormal bundle that passes between popliteal artery and vein. These are crucial signs for the diagnosis of PVES. The present study found that $83 \%(25 / 30)$ of the cases increased the distance between the popliteal artery and the popliteal vein, which was consistent with surgical results, suggesting high ultrasound sensitivity. This included six patients with bilateral popliteal vessel involvement. Collins PS, et al., [15] reviewed about $22 \%$ to $67 \%$ of the bilateral cases. Duplex scanning examination associated with pulsed Doppler offers a more precise scope of the popliteal fossa. This modality makes it possible to identify PVES by showing a popliteal artery may take abnormal course or an anatomical aberrant composition between the popliteal artery and vein that increases the distance separating the two vessels [16]. Separation of the arteries and veins can be shown on the ultrasound because the popliteal artery and popliteal vein move in a left-right direction, even if there is an arterial occlusion.
Popliteal vascular ultrasonography on the affected side shows varied degrees of vascular structure pathology, including thickening, lumen stenosis, and occlusion. When the popliteal artery is complicated by aneurysm, ultrasound examination can clearly find local thickening and aneurysmal dilation of the artery lumen. Even if the aneurysm is full of thrombosis, the aneurysm diagnosis can be confirmed. Duplex ultrasound therefore only shows the morphological structure of popliteal vessels, but also provides hemodynamic information [17]. It is a non-invasive method that might prove to be of great value in the diagnosis of PVES.

Provocative maneuvers can provide important information for early diagnosis of PVES. This type of examination allows real-time, dynamic observation of changes in popliteal vessel blood flow before and after the experiment, as well as demonstrates that compression was not detected in the neutral position $[4,18]$. The presence of entrapment should be highly suspected when the popliteal artery or vein are compressed. The popliteal vessels usually show signs of narrowing or lumen occlusion and have positive peak systolic velocity results. Therefore, close attention must be paid to the examination 

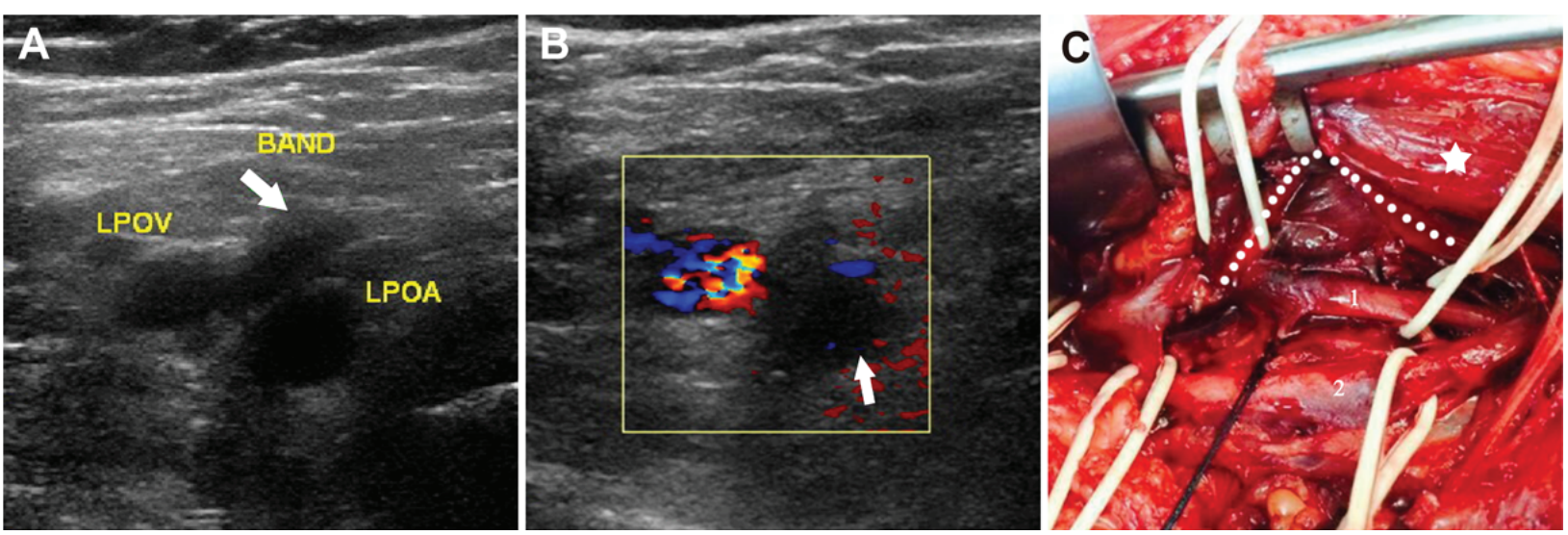

Figure 4: Left popliteal artery entrapment syndrome in 43-year-old man who presented with intermittent claudication of left calf and left foot coldness for three months (PVES type III). A: Ultrasound images show Left Popliteal Artery (LPOA) and Left Popliteal Vein (LOPV) widening. Left popliteal artery is entrapped by aberrant accessory muscle slip (arrow) of the medial head of left gastrocnemius muscle, which passes between the popliteal artery and vein; B: Left popliteal artery occlusion due to thrombosis. Color Doppler image shows that lumen is filled with hypoechoic thrombosis (arrow) and that popliteal artery was not filled with blood; C: Operation photography demonstrates popliteal artery (1) is entrapped by aberrant accessory muscle slip (dotted line) of the medial head of gastrocnemius muscle (star) that passes between the popliteal artery (1) and vein (2).
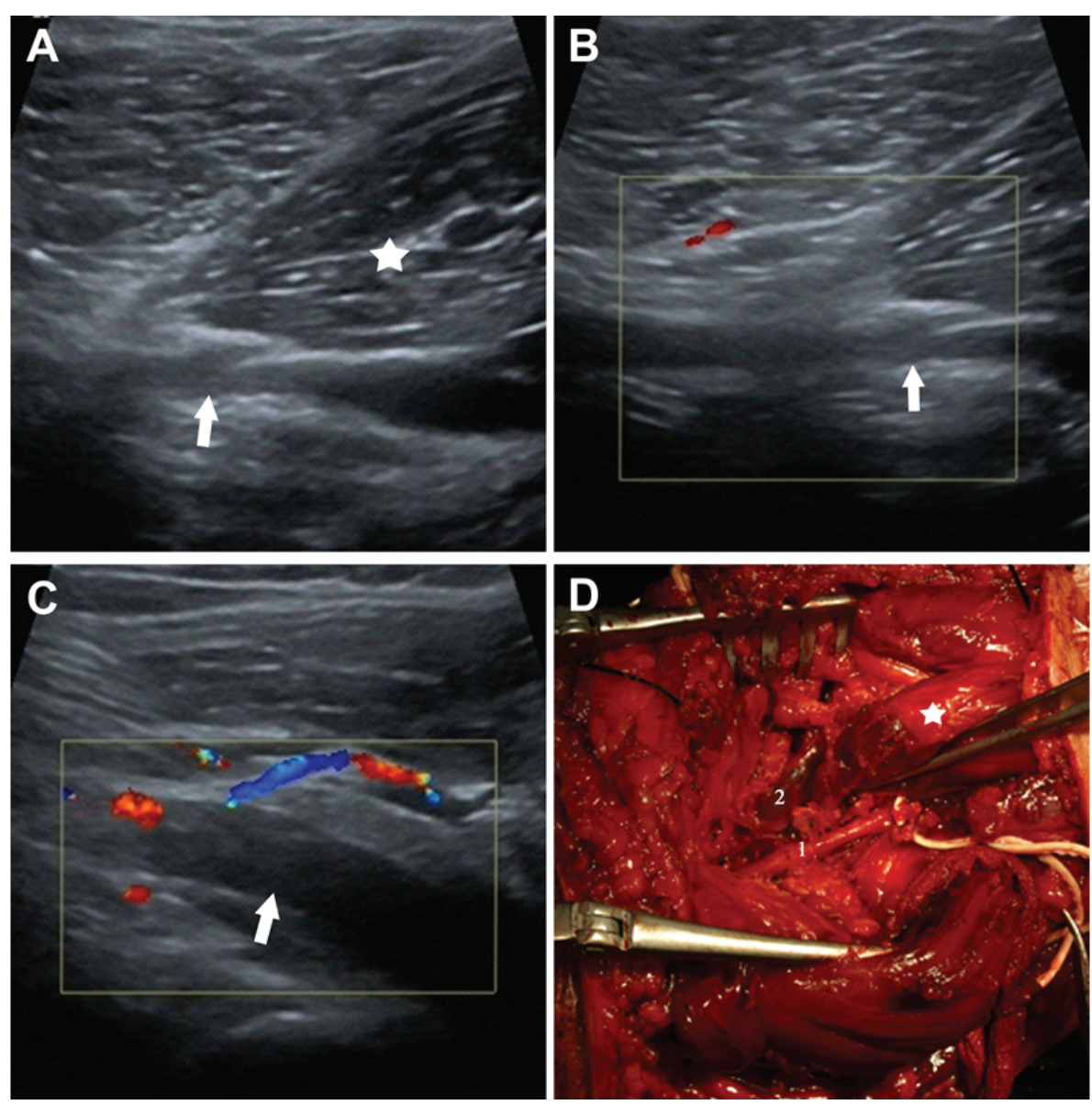

Figure 5: Right popliteal vascular entrapment syndrome in a 34-year-old man who presented with intermittent claudication of right calf for six months (PVES type II). A: Ultrasonic longitudinal section image shows the right popliteal artery (arrow) is entrapped by the laterally originated medial head of gastrocnemius muscle (star); B: Color Doppler image shows segmental stenosis of right popliteal artery (arrow) not filling with blood; C: Note that due to thrombosis (arrow), lumen is filled with medially echoic thrombosis and middle and distal segments of popliteal artery are occluded; D: Operation photography reveals the right popliteal artery (1) is entrapped by medial head of gastrocnemius muscle (star) that passes between the popliteal artery (1) and vein (2).

Citation: Zhong H, Liang Y, Shao G, Qi H, Zhao Y, et al. (2021) Doppler Ultrasound Evaluation of Patients with Popliteal Vascular Entrapment Syndrome. J Heart Health 7(2): dx.doi.org/10.16966/2379-769X.162 
Table 3: Ultrasound provocative maneuvers test findings for three cases with PVES.

\begin{tabular}{|c|c|c|}
\hline Case & $\begin{array}{l}\text { At rest } \\
\text { (in the neutral position) }\end{array}$ & $\begin{array}{l}\text { Provocative test } \\
\text { (in the plantar flexed position) }\end{array}$ \\
\hline Case 1 & Normal flow & $\begin{array}{l}\text { 1. Popliteal arterial stenosis } \\
\text { 2. Peak systolic blood velocity increased significantly in popliteal artery }\end{array}$ \\
\hline Case 2 & Normal flow & Popliteal arterial occlusion \\
\hline Case 3 & Normal flow & $\begin{array}{l}\text { 1. Popliteal arterial stenosis } \\
\text { 2. Peak systolic blood velocity increased significantly in popliteal artery } \\
\text { 3. Tibiofibular stem Doppler blood velocity was decreased }\end{array}$ \\
\hline
\end{tabular}

At rest, in a neutral position; Provocative test, in plantar flexed position

Table 4: Doppler ultrasound findings for patients with PVES in neutral position.

\begin{tabular}{|l|c|c|c|c|}
\hline \multicolumn{1}{|c|}{ Combined anomaly } & $\begin{array}{c}\text { Vascular stenosis } \\
\text { (N) }\end{array}$ & $\begin{array}{c}\text { Vascular occlusion } \\
\text { (N) }\end{array}$ & $\begin{array}{c}\text { Post-stenotic } \\
\text { aneurysm (N) }\end{array}$ & $\begin{array}{c}\text { Normal flow } \\
\text { (N) }\end{array}$ \\
\hline Gastrocnemius medial head anomaly & 10 & 13 & 2 \\
\hline No anatomical abnormalities & & 2 & 3 \\
\hline Total (N) & 10 & 15 & 25 \\
\hline
\end{tabular}

$\mathrm{N}$, number of limbs
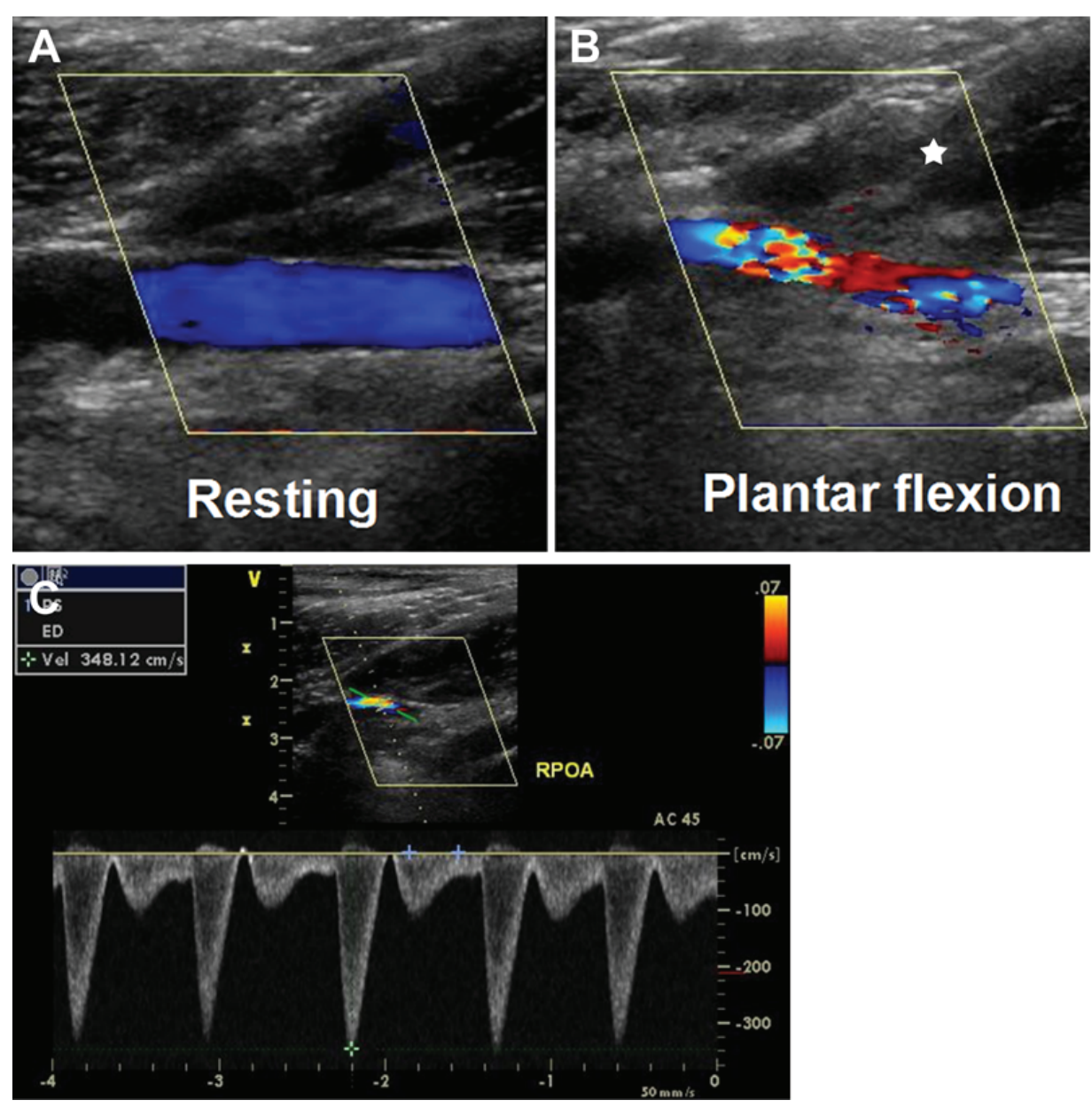

Figure 6: Right popliteal artery entrapment syndrome in 18-year-old man who presented with calf swelling and foot coldness in right lower extremity after physical activity. A: Color Doppler image of right foot in neutral position reveals right popliteal artery with normal flow; B: Color Doppler image of right foot in plantar flexion position shows segmental stenosis of right popliteal artery. Right popliteal artery is entrapped by medial head of gastrocnemius muscle (star); C: Doppler spectrum shows that peak systolic blood velocity increased significantly and more than doubled compared to its value prior to provocative maneuvers. 
of plantar flexion. Three examined cases had no abnormality in the neutral position, but popliteal vessels appeared to be narrowed or occluded during the provocative maneuvers, which was helpful for clinicians to consider the possibility of PVES. It is worth noting that for patients with complete popliteal artery occlusion, there is no blood flow signal in the popliteal artery during color Doppler examination. Thus, provocative maneuver test is applicable to patients without popliteal artery occlusion.

However, in two cases no popliteal fossa malformations were detected by ultrasound and the imaging was not specific. In this group, there were two cases with lower extremity atherosclerosis, with one 58-year-old male with history of left lower limb paresthesia for three years and claudication for four months. Ultrasound imaging found left lower limb artery atherosclerotic plaques and popliteal artery thrombosis. This patient underwent computed tomographic angiography, which showed that the left popliteal artery was entrapped by the medial head of the gastrocnemius which rose more laterally than the normal. Another case included a 55-year-old male who complained about symptoms of lower limb ischemia, such as swelling of the right leg and cold right foot for six months. Ultrasound suggested atherosclerosis of right lower extremities. The popliteal artery and tibiofibular stem Doppler blood flow velocity was decreased. The operation demonstrated compression of the right popliteal artery with a fibrous band that attached to the intercondylar notch after crossing the popliteal artery. Ultrasound examination in the above two patient's only diagnosed lower limb atherosclerosis and failed to find popliteal vascular depression. Thus, even though ultrasound scanning can show arterial lesions in older patients affected by atherosclerosis, pathological and etiological analysis still needs to combine clinical data and other imaging examinations. Digital subtraction angiography can clearly show the anatomical features of arterial injury. Seven cases (7/24) underwent DSA examination, which can show occlusion, deviation, aneurysm, and dynamic stenosis of the popliteal artery. However, DSA is a radiative and invasive test. DSA findings of PVES don't provide visualization of the popliteal artery and the surrounding structures.

For patients with PVES, drug therapy cannot fundamentally treat the disease, and surgery management is the most effective treatment for the disease. Currently, it is believed that patients with PVES should undergo a surgical treatment as soon as they are diagnosed. The principle of surgery is that $[19,20]$ when the popliteal artery is not injured, the medial head of the gastrocnemius muscle causing popliteal artery constriction or abnormal accessory muscle bundles and tendons can be released. In case of popliteal artery injury, endarterectomy and venous patch repair can be used. When popliteal artery is completely occluded, great saphenous vein autograft or artificial vascular bypass can be performed [21]. Doppler ultrasound can reveal locations of the saphenous vein and provide accurate positioning for surgery (Figures $3 \mathrm{~d}$ and $3 \mathrm{e}$ ). In addition, patients with popliteal artery and saphenous vein patency during postoperative follow-up can be evaluated using ultrasonography [3]. For some patients with popliteal artery compression and proximal side aneurysmal dilatation, ultrasound images can indicate the scope of aneurysms and mark them on the body surface, facilitating clinical selection of surgical methods.

Liu Y, et al. suggested that Doppler ultrasound is a simple, rapid, non-invasive procedure that should be used routinely in the evaluation of individuals with suspected PVES [3]. However, the use of Doppler sonography is limited by its small window, operator proficiency, probe pressure, and lack of classification accuracy. Therefore, further imaging studies are required for patients with suspicion of entrapment, even if Doppler results are normal.

\section{Conclusion}

Doppler ultrasound may have a high diagnostic rate of depicting clinical characteristics in patients with abnormal peripheral artery disease. PVES can be diagnosed by ultrasonography if the distance between the popliteal artery and vein is widened and medial displacement of the popliteal artery and abnormal muscle tissue between the blood vessels are present. It is a non-invasive and simple peripheral vascular examination tool. Provocative maneuvers allow direct observation of the popliteal vessels while they become entrapped. Simultaneous monitoring of the effects on vascular blood flow may help diagnose the syndrome. In conclusion, these results suggest that Doppler ultrasound can be used as a primary screening examination modality in PVES.

\section{Acknowledgements}

We thank International Science Editing (http://www. internationalscienceediting.com) for editing this manuscript.

\section{Compliance with Ethical Standards}

The retrospective study has been approved by the institutional ethics committee of the Second Hospital of Shandong University (KYLL-2019(LW)025), and the need for informed consent was waived.

\section{Conflict of Interest}

The authors declare that they have no conflicts of interests.

\section{References}

1. Rich NM, Collins GJ Jr, McDonald PT, Kozloff L, Clagett GP, et al. (1979) Popliteal vascular entrapment. Its increasing interest. Arch Surg 114: 1377-1384.

2. Levien LJ, Veller MG (1999) Popliteal artery entrapment syndrome: more common than previously recognized. J Vasc Surg 30: 587-598.

3. Liu $Y$, Sun $Y$, He X, Kong $Q$, Zhang $Y$, et al. (2014) Imaging Diagnosis and Surgical Treatment of Popliteal Artery Entrapment Syndrome: A Single-Center Experience. Ann Vasc Surg 28: 330-337.

4. Altintas U, Helgstrand UV, Hansen MA, Stentzer KF, Schroeder TV, et al. (2013) Popliteal artery entrapment syndrome: ultrasound imaging, intraoperative findings, and clinical outcome. Vasc Endovasc Surg 47: 513-518.

5. Williams C, Kennedy D, Bastian-Jordan M, Hislop M, Cramp B, et al. (2015) A new diagnostic approach to popliteal artery entrapment syndrome. J Med Radiat Sci 62: 226-229.

6. Nuffer Z, Rupasov A, Bekal N, Murtha J, Bhatt S (2017) Spectral Doppler ultrasound of peripheral arteries: a pictorial review. Clin Imaging 46: 91-97.

7. Haimovivi $H(1984)$ Vascular surgery: principles and techniques. $2^{\text {nd }}$ Edition, Appleton-Century-Crofts, United Kingdom 557-567.

8. Gerkin TM, Beebe HG, Williams DM, Bloom JR, Wakefield TW (1993) Popliteal vein entrapment presenting as deep venous thrombosis and chronic venous insufficiency. J Vasc Surg 18: 760-766.

9. Henry HT, Szolomayer LK, Sumpio BE, Sutton KM (2018) Popliteal Artery Entrapment Syndrome: Bilateral Lower Extremity Involvement. Orthopedics 41: e295-e298.

10. Hai Z, Guangrui S, Yuan Z, Zhuodong X, Cheng L, et al. (2008) CT angiography and MRI in patients with popliteal artery entrapment syndrome. AJR Am J Roentgenol 191: 1760-1766. 
11. Hwang JY (2017) Doppler ultrasonography of the lower extremity arteries: anatomy and scanning guidelines. Ultrasonography 36 : 111-119.

12. Rangankar VP, Taori KB, Mundhada RG, Rewatkar AD (2016) Accuracy of Common Femoral Artery Doppler Waveform Analysis in Predicting Haemodynamically Significant Aortoiliac Lesions. J Clin Diagn Res 10: TC26-TC28.

13. Mohler ER 3rd, Bundens W, Denenberg J, Medenilla E, Hiatt WR, et al. (2012) Progression of asymptomatic peripheral artery disease over 1 year. Vasc Med 17: 10-16.

14. Miao Z, Chuang C, Qianyi Q, Wu C (2016) Ultrasound in diagnosis of anatomical variation of anterior and posterior tibial arteries. Med Ultrason 18: 64-69.

15. Collins PS, McDonald PT, Lim RC (1989) Popliteal artery entrapment: an evolving syndrome. J Vasc Surg 10: 484-490.

16. Rosset E, Hartung O, Brunet C, Roche PH, Magnan PE, et al. (1995) Popliteal artery entrapment syndrome: anatomic and embryologic bases, diagnostic and therapeutic considerations following a series of 15 cases with a review of the literature. Surg Radiol Anat 17: 161169, 23-27.
17. Moneta GL (2020) Tibial artery velocities in the diagnosis and followup of peripheral arterial disease. Semin Vasc Surg 33: 65-68.

18. Alvarez Rey I, Alvarez Rey G, Alvero Cruz JR, Jimenez Diaz JF, Alvarez Bustos G (2004) Popliteal artery entrapment syndrome in an elite rower: sonographic appearances. J Ultrasound Med 23: 1667-1674.

19. Shahi N, Arosemena M, Kwon J, Abai B, Salvatore D, et al. (2019) Functional Popliteal Artery Entrapment Syndrome: A Review of Diagnosis and Management. Ann Vasc Surg 59: 259-267.

20. Fujimura N, Hosokawa K, Obara H, Igari K, Akamatsu D, et al. (2021) Incidence, diagnosis and treatment of popliteal artery entrapment syndrome in current vascular practice in Japan. Cardiovasc Interv Ther 36: 506-513.

21. Lavingia KS, Dua A, Rothenberg KA, Fredericson M, Lee JT (2019) Surgical management of functional popliteal entrapment syndrome in athletes. J Vasc Surg 70: 1555-1562. 